\title{
Effects of Dietary Supplement Containing Melatonin on Reproductive Activity in Male Golden Hamsters
}

\author{
tDonchan Choi \\ Dept. of Life Science, College of Environmental Sciences, Yong-In University, Yongin 17092, Korea
}

\begin{abstract}
Melatonin is a pineal hormone that is synthesized and released at night under the light and dark cycles of a day. Its effects on the reproductive activities have well been established by the administration through various routes in photoperiodic animals. It was also identified in plants and named phytomelatonin. The capacity of the phytomelatonin was investigated in this investigation whether it affects the reproductive function in male golden hamster. As expected, animals housed in long photoperiod (long photoperiod, LP>12.5 hours of lights in a day) had large testes and animals kept in short photoperiod ( $\mathrm{SP} \leqq 12.5$ hours of lights in a day) showed remarkably reduced testes. The dietary supplement with melatonin itself induced the complete involution of testes. Pistachios that were reported to contain a large amount of melatonin demonstrated no effects at all in male golden hamsters. These results suggest that dietary supplement containing melatonin-rich foodstuff used in this investigation may not be enough to affect the reproductive endocrine system in male golden hamsters.
\end{abstract}

Key words : Dietary supplement, Melatonin, Reproductive function, Golden hamster

\section{INTRODUCTION}

Melatonin (N-acetyl-5-methoxytryptamine) is a hormone secreted from the pineal gland. It is synthesized and released largely at night under the regular cycle of light and dark of a day, via a multi-step pathway from its precursor the amino acid tryptophan taken up from the circulating blood vessels (Rollag et al., 1980; Sugden, 1989). The tryptophan is converted by the four enzymes, including a critical enzyme serotonin- $\mathrm{N}$-acetyl transferase, to produce melatonin (Arnao et al., 2018).

This indoleamine was first isolated as a neuro-hormone in the bovine pineal gland as early as 1958 (Lerner et al., 1958). It was also identified in plants in 1995 and named phytomelatonin in 2004 (Dubbels et al., 1995; Hattori et al., 1995; Blask et al., 2004; Paredes et al., 2009; Aguilera et al., 2016). Melatonin and phytomelatonin are structurally the identical molecule, but melatonin refers to animal origin and the term phytomelatonin to plant origin. The findings raised an interest in investigating the potential effects of plant phytomelatonin on the reproductive activity of small animals, among the diverse capacities reported so far, particularly through the edible plant diet (Choi, 2013b; Bonomini et al., 2018; Jiki et al., 2018).

In relation to the reproductive function, the influence of melatonin was widely investigated in small mammals, particularly the seasonal breeding animals whose reproductive activities are limited to a particular time of a year (Reiter, 1980; Stetson \& Watson-Whitmyre, 1984; Choi, 2001). One of the animals is golden hamsters inhabiting in the

\footnotetext{
Manuscript received April 7, 2019, Received in revised form April 25, 2019, Accepted April 2, 2019

${ }^{\dagger}$ Corresponding Author : Donchan Choi, Dept. of Life Science, College of Environmental Sciences, Yong-In University, Yonginsi 17092, Korea. Tel: +82-31-8020-2781, Fax: +82-31-8020-2886, E-mail: dcchoi@yongin.ac.kr

This is an Open Access article distributed under the terms of the Creative Commons Attribution Non-Commercial License (http:// creative-commons.org/licenses/by-nc/3.0) which permits unrestricted non-commercial use, distribution, and reproduction in any medium, provided the original work is properly cited.
} 
temperate regions. They breed actively around summer of favorable seasons and their sexual activities are completely degenerated in winter of severe climate.

The main factor determining the sexual functions has been verified as the photoperiod that is a length of light in a day, which is surmised that the animals can predict possibly the annual cycle in a period of a year (Choi \& Lee, 2012). The alternate changes of seasonal reproductive function in the hamsters can be reproduced in the laboratory facility equipped with the artificial lighting regime. When the sexually mature male hamsters are transferred to short photoperiod (SP, equal to and less than 12 hours of lights in a day), they lose generative activities, showing small testes and no functional spermatozoa in testes (Stetson \& Watson-Whitmyre, 1986; Choi, 2012). If the length of lighting in a day is set to more than 12.5 hours (long photoperiod, LP) imitating summer season, the generative functions are actively recommenced and maintained afterward (Stetson \& Watson-Whitmyre, 1984). The photoperiodic information is relayed to the pineal gland where melatonin is synthesized and released. Melatonin is generated at only night due to the activated enzyme of serotonin-Nacetyl transferase, as mentioned above. In natural environment, the period of dark of a day is lengthened as the season progresses toward winter. The animals expose to the elongated period of melatonin which impacts on the reproductive endocrine system.

The melatonin has been considered to indirectly act on gonadotropin releasing hormone $(\mathrm{GnRH})$ neurons in the hypothalamus of the brain. There are numerous reports that exogenous administrations of melatonin resulted in reproductive regression (Reiter, 1980; Stetson \& WatsonWhitmyre, 1984). Melatonin has been injected or infused on a daily basis, or implanted by using capsules (Stetson \& Tay, 1983; Stetson \& Watson-Whitmyre, 1986; Hong \& Stetson, 1988; Maywood et al., 1991; Grosse et al., 1993; Choi, 2013a). On the basis of the impact of melatonin, the effects of phytomelatonin included in the natural foodstuff were investigated. The pistachio was chosen by the description that it contained lots of phytomelatonin (Oladi et al., 2014; Meng et al., 2017).

In the present investigation, the effects of diet supplemented with melatonin itself or melatonin-rich foodstuff pistachio were examined on the testicular activity of mature male golden hamsters.

\section{MATERIALS AND METHODS}

\section{Animals}

Mature male golden hamsters (Mesocricatus auratus) weighing around $100 \mathrm{~g}$ were used for this study. They were housed in plastic cages under LP conditions of light and dark cycles (light of $14 \mathrm{~h}$ : darkness of $10 \mathrm{~h}$ ) with an ambient temperature of $22 \pm 1^{\circ} \mathrm{C}$. Reproductive activities of these hamsters are always active in the LP photoperiod. They were provided with reconstructed feed supplemented with commercial melatonin or powdered pistachio (natural products), and tap water ad libitum. The condition of management of animals was approved by the Yong-In University Institutional Animal Care and Use Committee (YUIACUC-2017-04).

\section{Preparation of dietary supplement}

\section{1) Feed complemented with melatonin itself}

The melatonin that was available commercially as tablets was used (CVS Health, USA). They were milled into fine powder. Whereas the normal standard laboratory mouse chow was moisturized with edible water. On the next day, the wet chow was crushed into small pieces, spreaded in plates, and were allowed to make half-dried chow by drying at $60^{\circ} \mathrm{C}$ for 6 hours in the food dryer. The control feed (vehicle), which did not contain melatonin element, was reconstructed with a mixture of the half-dried chow prepared above and the normal flour of one-third of dried standard laboratory mouse chow in weight. The experi- 
mental feed was mixed half-dried chow with the powdered melatonin at a particular concentration, that was previously mixed with proper amount of flour. The materials were reconstructed to make the feed pellets. The animals were provided with $600 \mu \mathrm{g}$ that was the high concentration group. The low concentration group was received one tenth of the high concentration group. The concentrations were determined enough to induce the testicular regression in male golden hamsters.

\section{2) Feed complemented with melatonin-rich pistachio}

The standard laboratory mouse chow was prepared as above and became half-dried chow. Whereas the unseasoned pistachios were purchased from the local market in Korea. They were ground with mixer machine and yielded the fine powder. The control feed (vehicle), which did not contain pistachio substance, was prepared mentioned above. The experimental feed of high concentration of pistachio substance was a blend of the half-dried chow and the normal flour of one-third of dried standard laboratory mouse chow in weight supplemented previously with powdered pistachio. The experimental feed of low concentration of pistachio substance was prepared like above except for one tenth of the pistachio substance used in high concentration. The melatonin (also called phytomelatonin) was reported to be included $200 \mu \mathrm{g}$ per $\mathrm{g}$ of dried pistachio weight (Oladi et al., 2014; Meng et al., 2017). The reconstructed feed was prepared to contain $50 \mu \mathrm{g}$ of phytomelatonin per $\mathrm{g}$ of the reconstructed feed. The mingled material was reconstructed to make the feed pellets. The pellets were dried as mentioned above. The animals in control group received the vehicle and the experimental animals received the reconstructed feed containing $600 \mu \mathrm{g}$ of melatonin per animal at high concentration and $60 \mu \mathrm{g}$ of melatonin per animal at low concentration.

\section{Photoperiod treatment and feeding}

The mature golden hamsters were housed in an envi- ronment set at LP or SP lighting regimes. The control animals were maintained in LP or SP and received vehicle, the reconstructed feed without pistachio or melatonin. All the experimental animals were housed in LP. They received the assigned reconstructed feed in the evening for 56 consecutive days. During the experimental period the body weights were measured at beginning and the end to observe any unusual behavior.

\section{Determination of testicular mass}

The testicular volumes were measured by laparotomy at a given time points. The hamsters were momentarily anesthetized using diethyl ether with the aid of assistant helper. The skin overlying the scrotal sac was excised in a proper length, the testes within the scrotal sac were protruded, and the major axis and the minor axis of the testes were snugly measured in one-tenth of millimeter by vernier calipers. The skin excised was then sutured with autoclips. Only one testis between two was gauged at an indicated time to reduce any unwanted trauma. At the end of experiment both testes were excised, weighed, and compared to inspect any difference. This method reduces greatly the number of animals needed and thus is economic by reducing numbers of animals (Watson-Whitmyre \& Stetson, 1985).

\section{Statistical analysis}

Data were expressed as mean \pm SD. Statistical analysis was performed using Student's t-test. Differences were considered to be significant at $p<0.05$.

\section{RESULTS}

\section{Feed complemented with melatonin itself}

\section{1) Body weight}

During the period of experimental time of 8 weeks, the unusual behavior of hamsters was observed at a certain hour of the day to evaluate any abnormal aspects. The 
body weights were not changed abruptly (data not shown). As the time went by, there was no any particular aberrant action in animals treated with the reconstructed feed complemented with melatonin itself. Thus, melatonin was determined not to affect any bizarre behavior.

\section{2) Testicular mass with time}

The general changes of testicular mass of golden hamster who ingested the reconstructed feed supplemented with melatonin are shown in Fig. 1. At the end of 8 weeks experiment, all the animals except for the LP control animals showed very small testes that meant a complete testicular regression.

The testicular mass of individual animals in each group are shown in Fig. 2. The reconstructed feed complemented with melatonin itself induced ultimately the animals to be infertile. Throughout the experiment the LP control animals had large testes (Fig. 2A). The SP animals showed very small testes at the end of the experiment, as expected (Fig. 2B). The animals those received the reconstructed feed supplemented with melatonin exhibited very small testes in both low and high groups (Fig. 2C and 2D). Overall, the amount of melatonin used in this study resulted in testicular involution.

\section{3) Final testicular mass at the end of experiment}

At the end of experiment the actual weights of testes
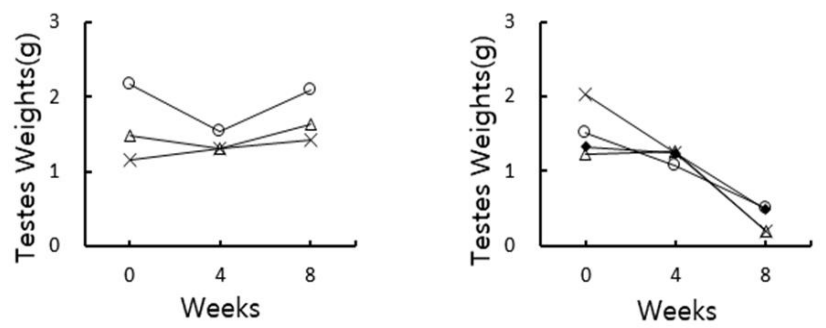

A. LP Cont

B. SP Cont

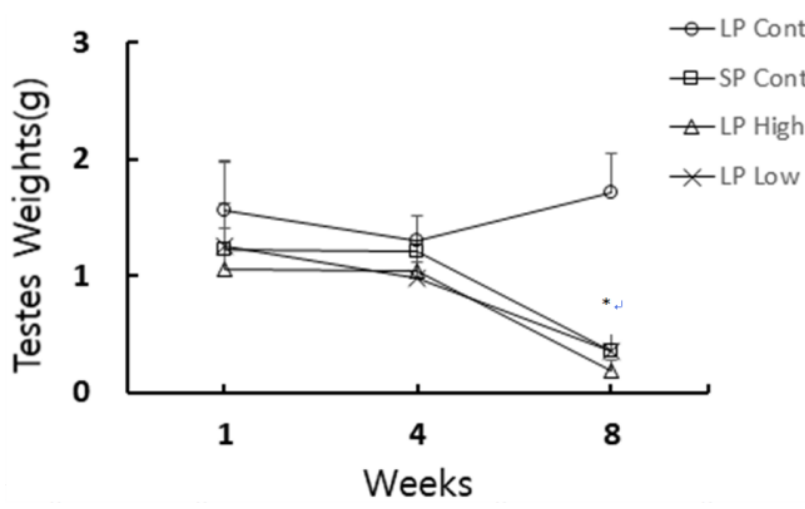

Fig. 1. The general changes of testicular mass of golden hamster ingested the reconstructed feed supplemented with melatonin itself. LP Cont and SP Cont: animals housed in LP and SP, respectively and treated with vehicle. LP Low and LP High: animals housed in LP and administered with low and high concentrations of melatonin in feed, respectively. ${ }^{*}$ Denotes statistically significant difference compared to the LP Cont $(p<0.05)$. LP, long photoperiod; SP, short photoperiod.

were individually weighed. The changes of testicular weights are shown in Fig. 3. There were no large differences between right and left testicles. Data were expressed as the paired testicular weights that were sum of both testicles in individual animal. The weights of the testes were maintained in LP animals but those in SP animals showed very small testes, which were statistically significant different $(p<0.05)$ when compared to the testes of the LP animals. Both the low and high concentration of melatonin treat-

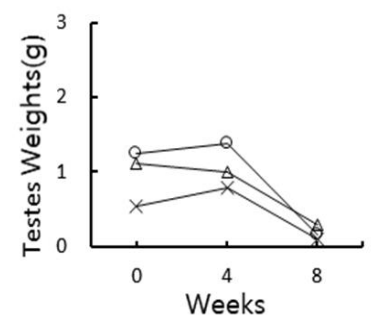

C. LP High

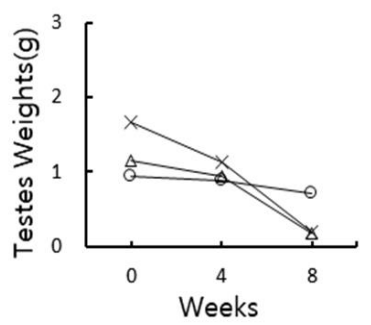

D. LP Low

Fig. 2. The changes of testicular mass of individual golden hamster who ingested the reconstructed feed supplemented with melatonin. LP Cont and SP Cont: animals housed in LP and SP, respectively and treated with vehicle. LP Low and LP High: animals housed in LP and administered with low and high concentrations of melatonin in feed, respectively. LP, long photoperiod; SP, short photoperiod. 


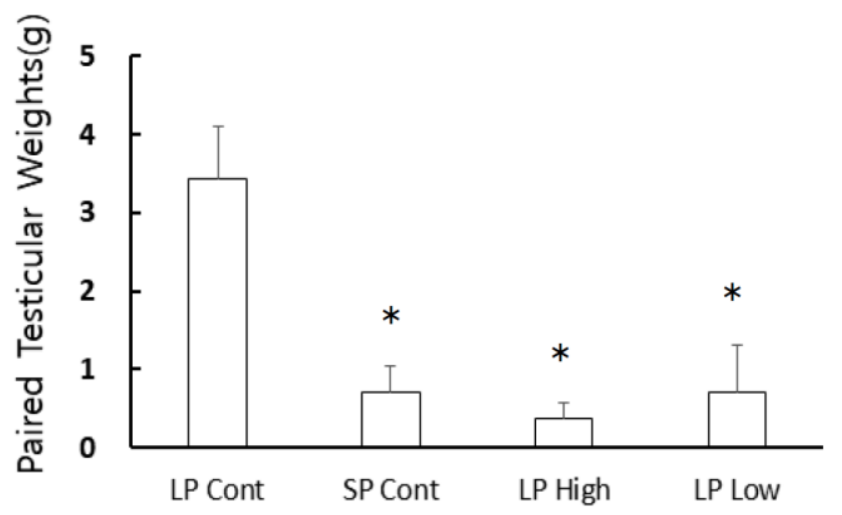

Fig. 3. Changes of actual testicular weights at the end of experiment. Note that animals administered with both low and high concentrations of melatonin in feed showed small testes as those housed in SP. LP Cont and SP Cont: animals housed in LP and SP, respectively and treated with vehicle. LP Low and LP High: animals housed in LP and administered with low and high concentrations of melatonin in feed, respectively. * Indicates statistical significance compared to LP Cont $(p<0.05)$. $\mathrm{n} \geqq 3$. LP, long photoperiod; SP, short photoperiod.

ments exhibited very small testes as in the SP control animals. The results mean that melatonin induced the testes to regress as the SP normally did.

\section{4) Changes of internal organs}

In order to examine any variations of internal organs, various organs were isolated and weighed at the end of the experiment (Table 1). The reproductive accessory organs, both epididymis and seminal vesicles, were apparently reduced in SP control animals and all the animals who ingested the feed supplemented with melatonin (Table 1). These results were consistent with the changes of the testes mentioned above. The weights of the other organs that were unrelated to the reproduction were not noticeably altered by the feed supplemented with melatonin.

\section{Feed complemented with melatonin-rich pistachio}

\section{1) Body weight}

Because the period of administration was long time of 8 weeks, the unusual behavior of hamsters was observed to evaluate any abnormal aspects during the whole experiment. As the time proceeded, there was no any particular aberrant action in animals treated with the feed recon-

Table 1. Changes of weights of various organs of the animals who ingested the feed supplemented with melatonin

\begin{tabular}{ccccc}
\hline \hline & LP Cont & SP Cont & LP High & LP Low \\
\hline Heart (g) & $0.43 \pm 0.134$ & $0.47 \pm 0.101$ & $0.43 \pm 0.066$ & $0.43 \pm 0.012$ \\
Kidney (g) & $0.50 \pm 0.056$ & $0.48 \pm 0.068$ & $0.47 \pm 0.054$ & $0.46 \pm 0.022$ \\
Liver (g) & $2.58 \pm 0.469$ & $2.58 \pm 0.579$ & $2.78 \pm 0.419$ & $2.75 \pm 0.273$ \\
Lung (g) & $0.69 \pm 0.178$ & $0.65 \pm 0.226$ & $0.66 \pm 0.155$ & $0.67 \pm 0.070$ \\
Spleen (g) & $0.09 \pm 0.033$ & $0.10 \pm 0.041$ & $0.10 \pm 0.026$ & $0.08 \pm 0.006$ \\
Seminal vesicle (g) & $1.65 \pm 0.297$ & $0.30 \pm 0.140^{*}$ & $0.19 \pm 0.075^{*}$ & $0.23 \pm 0.128^{*}$ \\
Epididymis (g) & $0.64 \pm 0.110$ & $0.12 \pm 0.117^{*}$ & $0.06 \pm 0.035^{*}$ & $0.13 \pm 0.092^{*}$ \\
\hline
\end{tabular}

Data are represented as the mean $\pm \mathrm{SD}(\mathrm{n} \geqq 3)$.

LP Cont and SP Cont: animals housed in LP and SP, respectively and treated with vehicle. LP Low and LP High: animals housed in LP and administered with low and high concentrations of the melatonin in feed, respectively.

* Denotes statistically significant difference compared to the LP Cont $(p<0.05)$.

LP, long photoperiod; SP, short photoperiod. 
structed with pistachio (data not shown). Thus, the amount of pistachio used in this experiment was concluded not to induce any bizarre behavior. The body weights of animals gradually increased from the beginning of the experiment (data not shown). The change of body weights during the entire experimental period of time was matched with the growth pattern.

\section{2) Testicular mass with time}

The general changes of testicular mass of golden hamster who ingested the reconstructed feed supplemented with melatonin-rich pistachio are shown in Fig. 4. The pistachio was reported to contain melatonin in high amount. At the end of 8 weeks experiment, all the animals including the LP control animals showed very large testes that meant a perfect preservation of reproductive function.

The changes of testicular mass of individual golden hamster who ingested the reconstructed feed with pistachio are shown in Fig. 5. All the animals had large testes, which indicated that pistachio had no effects on the reproductive activity in male golden hamsters. On the other hand, the SP control animals had low masses of testes (Fig. 1-3).

\section{3) Final testicular mass at the end of experiment}

At the end of experiment the actual weights of testes

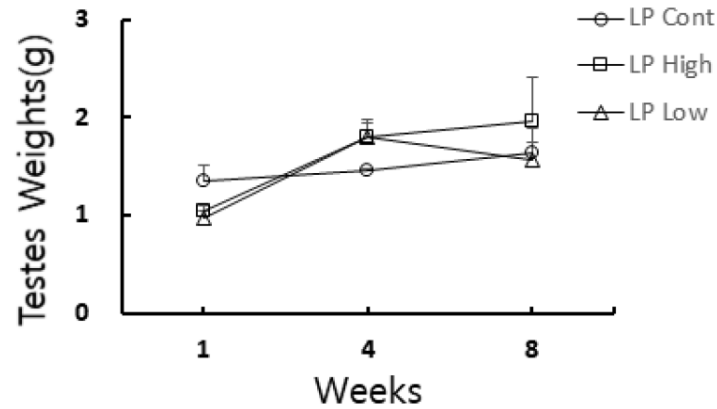

Fig. 4. The general changes of testicular mass of golden hamster who ingested the reconstructed feed supplemented with melatonin-rich pistachio. LP Cont: animals housed in LP and treated with vehicle. LP Low and LP High: animals housed in LP and administered with low and high concentrations of melatonin-rich foodstuff in feed, respectively. LP, long photoperiod.

were indeed individually weighed. The changes of testicular weights are shown in Fig. 6. The weights of the testes were maintained in LP animals at the end of experiment. On the other hand, those in SP animals were very small (Fig. 1-3). The low and high concentrations of pistachio treatments showed large testes, which means ineffectiveness on testicular activity.

\section{4) Changes of internal organs}

In order to examine any variations of internal organs,

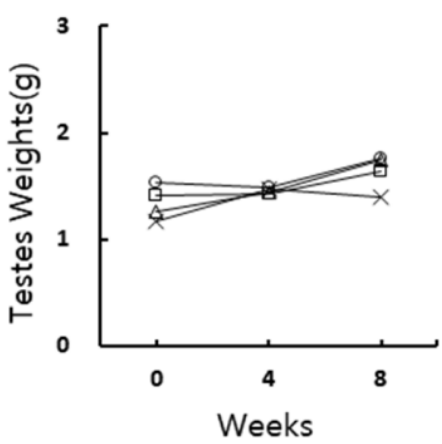

A. LP Cont

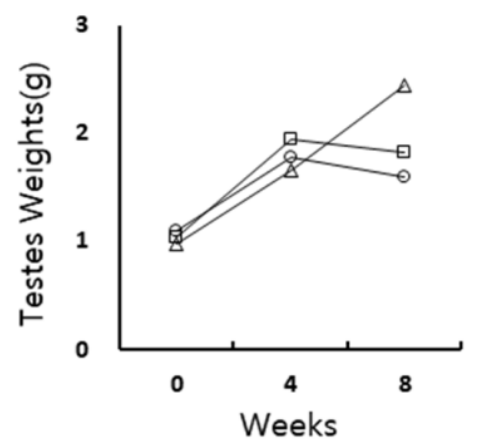

B. LP High

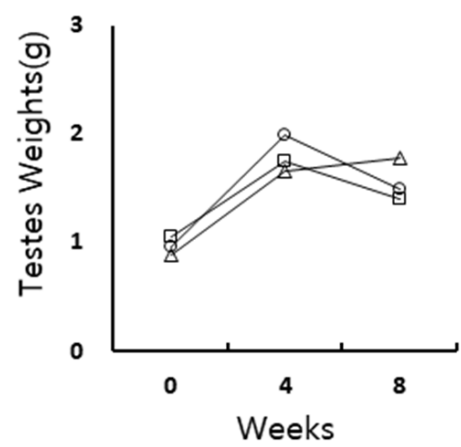

C. LP Low

Fig. 5. The changes of testicular mass of individual golden hamster who ingested the reconstructed feed supplemented with melatonin-rich pistachio. LP Cont: animals housed in LP and treated with vehicle. LP Low and LP High: animals housed in LP and administered with low and high concentrations of melatonin-rich foodstuff in feed, respectively. LP, long photoperiod; SP, short photoperiod. 


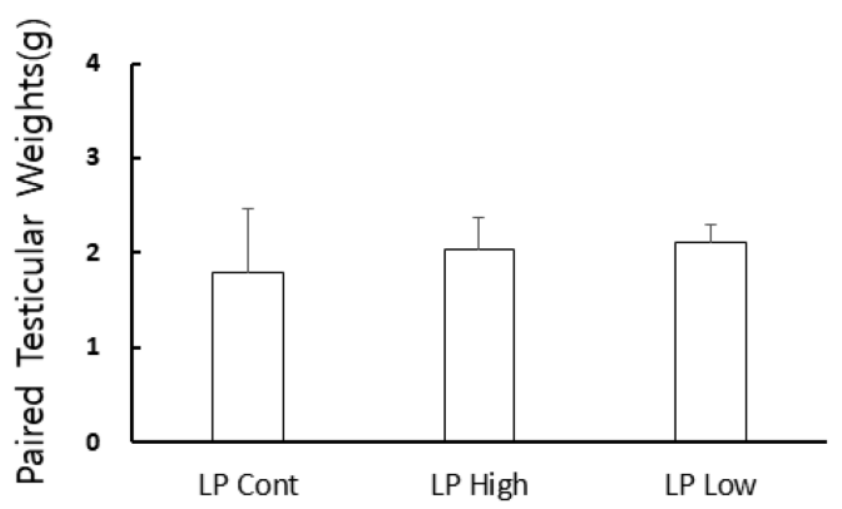

Fig. 6. Changes of actual testicular weights at the end of experiment. Note that animals administered with both low and high concentrations of melatonin-rich pistachio in feed showed large testes as those housed in LP. LP Cont: animals housed in LP treated with vehicle. LP Low and LP High: animals housed in LP and administered with low and high concentrations of melatonin-rich foodstuff in feed, respectively. LP, long photoperiod.

various organs were isolated and weighed at the end of the experiment (Table 2). Resultantly the weights of all the organs including the reproductive accessory organs were not noticeably altered by the pistachio substance admin- istration.

\section{DISCUSSION}

The present results obviously represent that the feed supplemented with melatonin induced degeneration of the reproductive function and the feed supplemented with melatonin-rich foodstuffs pistachio had no effects on the reproductive activities in male golden hamsters. This conclusion is proved by the conspicuous reductions of testicular mass and accessory sex organs in the animals who ingested the feed reconstructed with melatonin. Although the pistachio in which lots of melatonin were reported to be included was administered, the reproductive functions were maintained like the animals lodged in LP without lessened mass of reproductive organs.

Melatonin has well been established to involute the reproductive activity in the seasonal breeding animals when it was administered properly in a day. In golden hamsters, it has been steadily documented that the times by using injections, which caused the testicular regression, were

Table 2. Changes of weights of various organs of the animals who ingested the feed supplemented with melatoninrich pistachio

\begin{tabular}{cccc}
\hline \hline & LP Cont & LP High & LP Low \\
\hline Heart $(\mathrm{g})$ & $0.58 \pm 0.061$ & $0.67 \pm 0.058$ & $0.68 \pm 0.015$ \\
Kidney $(\mathrm{g})$ & $0.58 \pm 0.099$ & $0.57 \pm 0.029$ & $0.61 \pm 0.045$ \\
Liver $(\mathrm{g})$ & $4.88 \pm 0.430$ & $6.43 \pm 0.306$ & $5.97 \pm 0.297$ \\
Lung $(\mathrm{g})$ & $0.95 \pm 0.313$ & $1.17 \pm 0.115$ & $0.97 \pm 0.199$ \\
Spleen $(\mathrm{g})$ & $0.17 \pm 0.021$ & $0.15 \pm 0.006$ & $0.16 \pm 0.012$ \\
Seminal vesicle(g) & $1.16 \pm 0.354$ & $1.20 \pm 0.173$ & $0.90 \pm 0.235$ \\
Epididymis $(\mathrm{g})$ & $0.39 \pm 0.210$ & $0.60 \pm 0.105$ & $0.58 \pm 0.064$
\end{tabular}

Data are represented as the mean $\pm \mathrm{SD}(\mathrm{n} \geqq 3)$.

LP Cont: animals housed in LP and treated with vehicle. LP Low and LP High: animals housed in LP and administered with low and high concentrations of the melatonin-rich pistachio substance, respectively.

LP, long photoperiod. 
several hours before lights out (dusk) and one hour just before lights on (dawn) in a day (Stetson \& Tay, 1983). The injection of the other times, such as morning, early afternoon, and night, of a day were ineffective to invoke sexual involution in the animals. Thus the time that the animals received the feed was set at the evening in the present investigation, which was 4 hours before the lights turned off. The reconstructed feed that included melatonin itself caused the reproductive function to regress but the feed that supplemented with pistachio showed no effects on it.

The ranges of concentration of melatonin for the testes to involute were broad, depending on the routes of administration. No report has been announced that treatment of diet supplemented with melatonin or melatonin-containing foodstuffs influenced reproductive activities. The previous investigation used tens of micrograms of melatonin resulted in testicular involution (Stetson \& Tay, 1983). The dosage was referenced to reconstruct the feed that was used in this experiment. Resultantly the melatonin itself mixed in food presented regressing effects but supplements of melatonin-rich natural product pistachio showed no effects. The amounts of pistachio administered into the animals was expected to have the similar amount of melatonin, according to the previous finding (Oladi et al., 2014; Meng et al., 2017). Thus two sorts of administrations, supplemented with melatonin itself or melatonin-rich foodstuff, were speculated to result in similar output, testicular regression, but actual results were directly opposite. These results might be due to the discrepancy of absorption from the small intestine of the animals. Although melatonin was blended in the chow, it as a molecule could be absorbed easily and efficiently from the small intestine due to hydrophobic property of melatonin. Whereas the melatonin contained in pistachio might be absorbed inefficiently, somehow resulting in low level of melatonin in the blood stream than expected, which might not be enough to induce the reproductive involution. But it can not be ruled out that the more amount of pistachio or the decocted ex- tract could induce reproductive endocrine system and bring on the testicular regression.

The other feature to be considered is the entire period of administration. Golden hamsters housed in LP have constantly large testicular mass that implies sexually active functional activity (Choi \& Han, 2010; Choi \& Lee, 2012). But when the animals moved into SP, the testicular regression is occurred within the period of 8 weeks. The testicles became small which meant complete and momentary cessation of spermatogenesis. Normally 8 weeks required has been reported to be enough to testify the sexual regression. The experimental period of time applied in this investigation was 8 weeks, and the feed supplemented with melatonin caused sexual degeneration but the feed supplemented with melatonin-rich foodstuff did not. Thus the possibility that longer administration could alter the photoperiodic effects on the sexual function in this animal is reasonably low.

One of the methods to evaluate toxicity of long term administration is to measure any unnatural changes in the body weight. In the present investigation no sudden alterations in the body weights were observed in golden hamsters through the entire experiment. It means that the feed that animals ingested represented no general reproductive toxicity at the level of doses administered.

It has been substantiated that the reproductive activities of golden hamsters are regulated by $\mathrm{GnRH}$ as other mammals. And it has been found that the GnRH neurons in hypothalamus is indirectly affected by the melatonin, which is a major hormone secreted from the pineal gland (Choi, 2013a). The synthesis and release of melatonin is proportional to the length of nighttime, thus melatonin is secreted for expanded period of time in winter, which affects the reproductive neuroendocrine system in the golden hamsters. The hypothalamic-pituitary-gonadal axis (HPG axis) is responsible for initiation and maintenance of spermatogenetic activity. GnRH is produced and secreted to the pituitary that releases follicle stimulating hormone (FSH) 
and luteinizing hormne (LH). Both FSH and LH act on the testes. In the testes, LH stimulates the Leydig cells, located in interstitial tissue of seminiferous tubules. Then the cells produce and secrete testosterone. Simultaneously, FSH supports the function of sertoli cells, a mediator for effects of testosterone and exerts its effect on germ cells for successful spermatogenesis in seminiferous tubules.

The action mechanism of the melatonin could be speculated as follows no matter what feeds were used. The feed containing melatonin would be swallowed into the animal body and be absorbed in the gastrointestinal tract and then spreaded all over the body through the circulatory system. Thus melatonin could act on hypothalamus, ultimately on the cells generating $\mathrm{GnRH}$, resulting in reproductive degeneration. In the present investigation, the ingestion of diet supplemented with melatonin caused the testis to regress. But the feed supplemented with melatonin-rich foodstuff showed no effect at all in HPG axis of the male golden hamsters. The discrepancy could be due to the level of melatonin in blood. Phytomelatonin included in the feed containing pistachio could not be practically absorbed in the gastrointestinal tract. Or the action of melatonin could be disturbed by the other unknown compounds absorbed with phytomelatonin, as reported previously (Peuhkuri et al., 2012). Although the coffee beans included high concentration of melatonin, the levels of melatonin in the circulation following ingestion showed contradictory results, such as increase or decrease (Peuhkuri et al., 2012; Ramakrishna et al., 2012; Arnao \& Hernandez-Ruiz, 2018).

In conclusion, the present results imply that the ingestion of diet supplemented with melatonin itself affected the reproductive system and caused the testis to regress. But the feed supplemented with melatonin-rich foodstuff pistachio showed no effect at all in male golden hamsters. The discrepancy could be due to the level of melatonin in blood. Phytomelatonin in the feed could be interfered in the course of absorption in the gastrointestinal tract or disturbed by the other unknown compounds absorbed with melatonin.
Further investigation is required to find whether dietary supplementations could alter the reproductive function.

\section{ACKNOWLEDGEMENTS}

This research was supported by a research grant from Yong-In University (2017-03).

\section{REFERENCES}

Aguilera Y, Rebollo-Hernanz M, Herrera T, Cayuelas LT, Rodriguez-Rodriguez P, de Pablo AL, Arribas SM, Martin-Cabrejas MA (2016) Intake of bean sprouts influences melatonin and antioxidant capacity biomarker levels in rats. Food Funct 7:1438-1445.

Arnao MB, Hernandez-Ruiz J (2018) The potential of phytomelatonin as a nutraceutical. Molecules 23:238-256.

Blask DE, Dauchy RT, Sauer LA, Krause JA (2004) Melatonin uptake and growth prevention in rat hepatoma 7288CTC in response to dietary melatonin: melatonin receptor-mediated inhibition of tumor linoleic acid metabolism to the growth signaling molecule 13-hydroxyoctadecadienoic acid and the potential role of phytomelatonin. Carcinogenesis 25:951-960.

Bonomini F, Borsani E, Favero G, Rodella LF, Rezzani R (2018) Dietary melatonin supplementation could be a promising preventing/therapeutic approach for a variety of liver diseases. Nutrients 10:1135-1159.

Choi D (2001) Influence of melatonin on reproductive function in male golden hamsters. Dev Reprod 5:1-8.

Choi D (2012) The consequences of mutations in the reproductive endocrine system. Dev Reprod 16:235-251.

Choi D (2013a) Continuous melatonin attenuates the regressing activities of short photoperiod in male golden hamsters. Dev Reprod 17:111-119.

Choi D (2013b) Potency of melatonin in living beings. Dev Reprod 17:149-177.

Choi D, Han E (2010) The impacts of photoperiods on 
hypothalamic proteins in the reproductive activities of golden hamsters. Dev Reprod 14:185-197.

Choi D, Lee SH (2012) Neuroendocrine system in seasonal breeder: Focusing on the reproductive activity of male golden hamster. Dev Reprod 16:1-8.

Dubbels R, Reiter RJ, Klenke E, Goebel A, Schnakenberg E, Ehlers C, Schiwara HW, Schloot W (1995) Melatonin in edible plants identified by radioimmunoassay and by high performance liquid chromatography-mass spectrometry. J Pineal Res 18:28-31.

Grosse J, Maywood ES, Ebling FJ, Hastings MH (1993) Testicular regression in pinealectomized Syrian hamsters following infusions of melatonin delivered on non-circadian schedules. Biol Reprod 49:666-674.

Hattori A, Migitaka $\mathrm{H}$, Iigo $\mathrm{M}$, Itoh $\mathrm{M}$, Yamamoto $\mathrm{K}$, Ohtani-Kaneko R, Hara M, Suzuki T, Reiter RJ (1995) Identification of melatonin in plants and its effects on plasma melatonin levels and binding to melatonin receptors in vertebrates. Biochem Mol Biol Int 35:627634.

Hong SM, Stetson MH (1988) Termination of gonadal refractoriness in Turkish hamsters, Mesocricetus brandti. Biol Reprod 38:639-643.

Jiki Z, Lecour S, Nduhirabandi F (2018) Cardiovascular benefits of dietary melatonin: A myth or a reality? Front Physiol 9:528.

Lerner AB, Case JD, Takahashi Y, Lee TH, Mori W (1958) Isolation of melatonin, the pineal gland factor that lightens melanocytes. J Am Chem Soc 80:2587.

Maywood ES, Lindsay JO, Karp J, Power JB, Williams LM, Titchener L, Ebling FJ, Herbert J, Hasting MH (1991) Occlusion of the melatonin-free interval blocks the short day gonadal response of the male Syrian hamster to programmed melatonin infusions of necessary duration and amplitude. J Neuroendocrinol 3:331337.

Meng X, Li Y, Li S, Zhou Y, Gan RY, Xu DP, Li HB (2017) Dietary sources and bioactivities of melatonin. Nutri- ents 9:367-430.

Oladi E, Mohamadi M, Shamspur T, Mostafavi A (2014) Spectrofluorimetric determination of melatonin in kernels of four different Pistacia varieties after ultrasoundassisted solid-liquid extraction. Spectrochim Acta A Mol Biomol Spectrosc 132:326-329.

Paredes SD, Korkmaz A, Manchester LC, Tan DX, Reiter RJ (2009) Phytomelatonin: A review. J Exp Bot 60:5769.

Peuhkuri K, Sihvola N, Korpela R (2012) Dietary factors and fluctuating levels of melatonin. Food Nutr Res $56: 17252$.

Ramakrishna A, Giridhar P, Sankar KU, Ravishankar GA (2012) Melatonin and serotonin profiles in beans of Coffea species. J Pineal Res 52:470-476.

Reiter RJ (1980) The pineal and its hormones in the control of reproduction in mammals. Endocr Rev 1:109131.

Rollag MD, Panke ES, Trakulrungsi W, Trakulrungsi C, Reiter RJ (1980) Quantification of daily melatonin synthesis in the hamster pineal gland. Endocrinology 106 231-236.

Stetson M, Watson-Whitmyre M (1984) Physiology of the pineal and its hormone melatonin in annual reproduction in rodents. In: Reiter RJ (ed), The Pineal Gland. Raven Press, Boca Raton, FL, pp 109-153.

Stetson MH, Tay DE (1983) Time course of sensitivity of golden hamsters to melatonin injections throughout the day. Biol Reprod 29:432-438.

Stetson MH, Watson-Whitmyre M (1986) Effects of exogenous and endogenous melatonin on gonadal function in hamsters. J Neural Transm 21:55-80.

Sugden D (1989) Melatonin biosynthesis in the mammalian pineal gland. Experientia 45:922-932.

Watson-Whitmyre M, Stetson MH (1985) A mathematical method for estimating paired testes weight from in situ testicular measurements in three species of hamster. Anat Rec 213:473-476. 